\title{
ĐỀ XUẤT MÔ HÌNH CDIO KHÉP KÍN ĐỂ CẢI THIỆN KHẢ NĂNG KHỞI NGHIỆP CHO SINH VIÊN KHOA ĐIỆN - ĐIÊ̂N TỬ, ĐẠI HỌC DUY TÂN

\author{
Hà Đắc Bình ${ }^{(*)}$, Trương Văn Trương ${ }^{(*)}$, Trần Nhật Tân ${ }^{(* *)}$
} \\ (*) Thạc sĩ. Đại học Duy Tân. Email: hadacbinh@duytan.edu.vn. \\ (**)Tiến sĩ.Đại học Duy Tân.Email:trannhattan@duytan.edu.vn.
}

DOI: $\underline{10.37550 / t d m u . C F R / 2021.01 .142 ~}$

\section{Tóm tắt}

Sinh viên chuyên ngành Kỹ thuật Điện \& Điện tủ (EEE) có khả năng tạo ra một số sản phẩm thông qua việc họ tập dụa trên đồ án CDIO để khởi nghiệp. Tuy nhiên, ho thiếu khả năng đổi mói liên tục để đạt được hiệu quả khởi nghiệp. Trong quá trình thực hiện giảng dạy CDIO ơTrường Đại học Duy Tân, chúng tôi đã tích lũy được một số kinh nghiệm để giải quyết vấn đề này và thu được một số kết quả khả quan và sẽ được trình bày trong bài viết này. Đóng góp đầu tiên là một đề xuất về một mô hình dạy và học để nâng cao khả năng khởi nghiệp, cu thể là CDIO khép kín dựa trên mô hìn CDIO thông thương. Sau giai đoạn Vận hành (Operation), sinh viên được khuyến khích và đào tạo để tiếp tục hình thành ý tương móti để cải tiến hoạc tạo ra sản phẩm mới dụa trên ý tưởng truớc đó. Các vấn đề cải tiến bao gồm bổ sung tính năng, cải thiện thông số kỹ thuật, xem xét về chi phí bảo trì, v.v. Khung CDIO khép kín cho phép chúng tôi cải thiện khả năng đổi mới liên tục của sinh viên EEE để nâng cao sụ cạnh tranh của sản phẩm được họ tạo ra. Trong mô hình được đề xuất này, chúng tôi nhấn mạnh sụ đổi mới không ngùng để đáp ứng yêu cầu của người tiêu dùng. Đóng góp thứ hai của bài viết này là việc đánh giá khung đề xuất này dựa trên các sản phẩm được chấp nhận trên thị truờng. Chúng tôi đã điều tra thống kê sau năm năm thực hiện khung CDIO này trong khoa của chúng tôi tù̀ 2013-2014 đến 2017-2018 và kết quả khẳng định tính hiệu quả của mô hình đề xuất. Để làm rõ hiệu quả, chúng tôi cũng trình bày một trường hợp thực tế, đó là các sản phẩm nhà thông minh. Trong truoòng hợp đó, chúng tôi mô tả chi tiết quy trình áp dụng khung CDIO khép kín để nâng cao khả năng khởi động dựa trên các sản phẩm nhà thông minh. Chúng tôi sẽ thảo luận chi tiết hơn về các công trình của chúng tôi để giải quyết nhũng vấn đề này trong báo cáo đầy đu.

Tù̀ khóa: CDIO khép kín, khởi nghiệp, sáng tạo, sản phẩm thục tế

\section{1. Đặt vấn đề}

Với dân số lớn 90 triệu người và phần lớn là thanh niên, khoảng $40 \%$ trong số đó dưới 25 tuổi, Việt Nam trở thành thị trường tiềm năng lớn thứ ba ở Đông Nam Á. Nó mang lại cơ hội 
lớn cho các công ty khai thác thị trường mới nổi này. Nhận thức được vấn đề đó, chính phủ Việt Nam đã và đang khuyến khích tinh thần khởi nghiệp từ toàn xã hội trong những năm gần đây (www.startup.gov.vn). Đại học Duy Tân, cái nôi đào tạo nguồn nhân lực cho startup, cũng đã và đang triển khai việc cung cấp kiến thức khởi nghiệp cho sinh viên của trường. Để khởi nghiệp, chúng ta không chỉ phải có kiến thức về quản lý, marketing, kỹ năng mềm mà còn phải có kiến thức nhất định về sản phẩm, dịch vụ kinh doanh. Đặc biệt, để tăng khả năng cạnh tranh của các doanh nghiệp trẻ, chủ doanh nghiệp phải có sản phẩm sáng tạo, độc đáo (Adam Szirmai, 2011). Không ngừng đổi mới ý tưởng là chìa khóa cho các sản phẩm hoặc dịch vụ sáng tạo (Fei Bian, 2013). Tuy nhiên, việc trau dồi khả năng đổi mới trong lĩnh vực Kỹ thuật Điện \& Điện tử (EEE) trở nên trì trệ do việc giáo dục đại trà ở Việt Nam không có bất kỳ hoạt động nào cải thiện nào về vấn đề. Có nhiều công trình tập trung vào việc nâng cao khả năng đổi mới sáng tạo của học sinh. Ví dụ, trong nghiên cứu của Wang, Yangqin và cộng sự, tác giả đã trình bày phương pháp giảng dạy nhằm nâng cao khả năng đổi mới của sinh viên chuyên ngành tự động hóa. Bằng việc triển khai quan niệm đổi mới giáo dục, xây dựng nền tảng thực nghiệm, xây dựng đội ngũ giảng viên và xây dựng đội ngũ đổi mới sáng tạo, tư duy đổi mới, tinh thần đổi mới và khả năng đổi mới của học sinh đã được trau dồi. Trong công trình "Nghiên cứu hệ thống dạy học thực nghiệm dựa trên năng lực thực hành đổi mới” (Wu, Tong Q., v.v., 2013), một hệ thống dạy học thực nghiệm dựa trên năng lực thực hành đã được trình bày.

Đại học Duy Tân đã và đang triển khai chương trình khởi nghiệp từ năm 2009 và khung CDIO trong chương trình giáo dục EEE từ năm học 2011-2012. Khi đó, chúng tôi đã đề xuất một khung dạy và học mới cho nghiên cứu khoa học, đó là CDIE (Conceive Design - implement - Assessment) tồn tại song song với mô hình CDIO (Bình Đắc HA và v.v., 2017). Sự kết hợp giữa nghiên cứu khoa học và phương pháp giảng dạy CDIO cho phép chúng tôi nâng cao khả năng đổi mới của sinh viên EEE tại Duy Tân. Trong mô hình đề xuất đó, chúng tôi nhấn mạnh giai đoạn Đánh giá cho các đồ án. Công việc này đã giúp sinh viên biết cách đánh giá kết quả mới của dự án của họ. Tuy nhiên, tại thời điểm đó, startup kết hợp và $\mathrm{CDIO}$ vẫn chưa được coi là chìa khóa mở ra cánh cửa thị trường sản phẩm điện \& điện tử cho sinh viên EEE.

Từ năm học 2013-2014, khoa của chúng tôi đã đề xuất và triển khai một mô hình mới, đó là khung CDIO khép kín để nâng cao khả năng đổi mới và khởi nghiệp cho sinh viên. Sinh viên của EEE chỉ tập trung vào việc nguyên mẫu của họ có hoạt động hay không, nhưng họ không xem xét nhiều về sản phẩm của họ dành cho khởi nghiệp. Và họ cũng không cân nhắc về cách tiếp tục cải tiến sản phẩm của mình để đáp ứng các yêu cầu của thị trường. Để giải quyết những vấn đề này, chúng tôi tích hợp nội dung chính của EEE và kiến thức kinh doanh trong quá trình triển khai các khóa học CDIO.

\section{Mô tả mô hình CDIO khép kín}

Trong chương trình học của EEE, có năm khóa học CDIO, cụ thể là CDIO 1 - CDIO 5 , được phân bổ trong 4 năm rưỡi đào tạo. Mỗi khóa học đều có khối lượng đánh giá kết quả học tập khác nhau tùy thuộc vào mục tiêu hướng đến, chi tiết tại Bảng 1 . Ở CDIO 1 , chúng tôi tập trung vào các hoạt động trau dồi khả tư duy cho sinh viên, vì vậy chúng tôi đặt trọng lượng cho khâu Conceive cao hơn so với những hoạt động khác. Trong CDIO 2, 
chúng tôi đào tạo sinh viên cách thiết kế một sản phẩm và chúng tôi đưa ra tỷ lệ đánh giá về Design cao hơn những người khác. Tương tự, trong CDIO 3 và $\mathrm{CDIO} 4$, chúng tôi lần lượt nhấn mạnh khả năng Implement và Operation. Cuối cùng, trong $\mathrm{CDIO} 5$, chúng tôi định hướng mỗi nhóm thực hiện một đồ án tổng hợp. Mỗi khóa học kéo dài từ 2-3 tháng.

\begin{tabular}{|c|l|l|l|}
\hline STT & Tên khóa học CDIO & \multicolumn{1}{|c|}{ Chuẩn đầu ra } & \multicolumn{1}{c|}{ Tỉ lệ đánh giá } \\
\hline 1 & CDIO 1 & Conceive & C40\%+D20\%+I20\%+O20\% \\
\hline 2 & CDIO 2 & Design & C20\%+D40\%+I20\%+O20\% \\
\hline 3 & CDIO 3 & Implement & C20\%+D20\%+I40\%+O20\% \\
\hline 4 & CDIO 4 & Operate & C20\%+D20\%+I20\%+O40\% \\
\hline 5 & CDIO 5 & Conceive-Design-Implement-Operate & C25\%+D25\%+I25\%+O25\% \\
\hline
\end{tabular}

Để trau dồi khả năng khởi nghiệp, chúng tôi đã triển khai khung CDIO khép kín ở Đại học Duy Tân trong vài năm trở lại đây, như Hình 1 . Điểm bắt đầu của vòng lặp này là Conceive, giống với khung $\mathrm{CDIO}$ thông thường. Tuy nhiên, khung $\mathrm{CDIO}$ này được thử áp dụng trong 4.5 năm với vòng lặp khép kín cho các đội được chọn. Cụ thể, trong các lớp CDIO, chúng tôi chỉ áp dụng khung này cho một số nhóm sinh viên. Chúng tôi để họ quyết định có theo đuổi mục tiêu khởi nghiệp hay không. Nhóm theo đuổi mục tiêu khởi nghiệp được chỉ định điều hành một dự án kinh doanh. Trong dự án này, họ sẽ theo đuổi ý tưởng về dòng sản phẩm của EEE có thể cung cấp cho thị trường, chẳng hạn như nhà thông minh. Trong mỗi khóa học CDIO, chúng tôi chuyển sang khuôn khổ CDIO như đã đề cập ở trên. Tuy nhiên, nhóm này không cần phải thay đổi sản phẩm của họ từ khóa học này sang khóa học khác. Nói cách khác, họ chỉ tập trung vào một loại sản phẩm có thể bán trên thị trường. Loại sản phẩm này sẽ tiếp tục được thông qua và cải tiến trong năm khóa học CDIO như sau.

Trong khóa học CDIO 1, các sinh viên được yêu cầu điều tra thị trường về các sản phẩm trong lĩnh vực của EEE. Tiếp theo, họ đề xuất ý tưởng trong nhóm của mình và quyết định sản phẩm cuối cùng. Sau đó, họ thiết kế ý tưởng của mình bằng cách vẽ và xây dựng một nguyên mẫu đơn giản để thuyết phục khách hàng tiềm năng của họ. Cuối cùng, họ điều chỉnh ý tưởng của mình theo kết quả họ nhận được từ cuộc khảo sát.

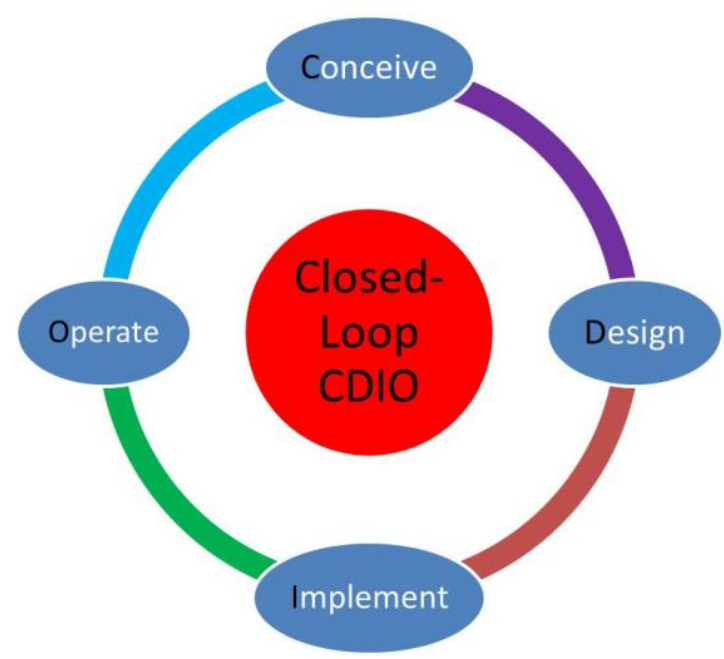

Hinh 1. Mô hìn CDIO khép kín (CL CDIO) 
Trong đồ án CDIO 2, các nhóm được chọn sẽ tiếp tục ý tưởng của mình nhưng họ nên tập trung vào khâu Thiết kế. Họ không chỉ tập trung vào thiết kế để chạy sản phẩm mà còn xem xét ngoại hình, giá thành, sự tiện lợi, v.v. để đáp ứng yêu cầu của thị trường. Tất nhiên, trong khóa học này, họ nên đề xuất ý tưởng để có được kết quả thiết kế tốt nhất. Họ cũng được yêu cầu sử dụng một số phần mềm và công cụ để thiết kế và mô phỏng sản phẩm của họ. Sau đó, họ hoàn thiện sản phẩm của mình bằng cách tạo ra nguyên mẫu phiên bản thứ hai. Cuối cùng, họ điều tra khách hàng về thiết kế của sản phẩm này và thảo luận trong nhóm của họ để tối ưu hóa thiết kế.

Trong đồ án CDIO 3, nhóm tìm kiếm ít nhất một khách hàng sẵn sàng sử dụng sản phẩm của họ. Sản phẩm này được cài đặt để sử dụng tại chỗ của khách hàng. Nhóm sẽ thiết kế quy trình sản xuất và lắp đặt, sau đó thực hiện cài đặt sản phẩm cho người dùng của họ. Cuối cùng, họ đánh giá hoạt động của sản phẩm dựa trên điều tra trải nghiệm người dùng.

Trong đồ án CDIO 4, nhóm được chọn tiếp tục đề xuất một số ý tưởng để cải thiện hệ thống, đồng thời duy trì hoạt động của sản phẩm bằng cách cải thiện một số chức năng hoặc thêm một chức năng mới theo yêu cầu của khách hàng. Cuối cùng, họ đánh giá hoạt động của sản phẩm của mình dựa trên điều tra trải nghiệm người dùng.

Trong đồ án CDIO 5, nhóm được chọn có thể thành lập công ty hoặc tham gia một tổ chức để điều hành dự án kinh doanh này. Các cố vấn cung cấp thêm kiến thức về quản lý kinh doanh cho họ và hướng dẫn họ cách điều hành dự án kinh doanh. Các sinh viên của nhóm được chọn tiếp tục đề xuất một số ý tưởng để đưa sản phẩm của mình ra thị trường.

Để đánh giá kết quả học tập của mỗi khóa học CDIO, chúng tôi tập trung vào các tiêu chí đánh giá chính sau:

Tiêu chí 1 . Tính mới và độc đáo của ý tưởng (ý tưởng, nguyên mẫu hoặc sản phẩm):

Đây là vấn đề chính của mỗi dự án, trong đó ý tưởng thúc đẩy dự án và tạo điều kiện cho việc tạo ra các sản phẩm mới hoặc nguyên mẫu là quan trọng nhất. Ngay cả khi một dự án không thành công, ý tưởng quảng bá nó vẫn có thể được đánh giá ở mức cao nếu nó được coi là hoàn toàn mới và nguyên bản. Tuy nhiên, việc đánh giá tính mới và tính độc đáo của các ý tưởng thường mang tính chủ quan. Do đó, Khoa Kỹ thuật Điện và Điện tử của chúng tôi đã thành lập Ban Đánh giá Dự án CDIO để xem xét tất cả các dự án CDIO trong bất kỳ học kỳ nào. Sinh viên được yêu cầu viết một báo cáo về ý tưởng dự án, sở thích của họ, ai sẽ nhận được lợi ích, sản phẩm dự kiến sẽ tồn tại bao lâu trên thị trường, v.v.

Tiêu chí 2. Cấu trúc lôgic của dự án:

Với ý tưởng dự án, trước hết, sinh viên sẽ cần xây dựng lộ trình cho dự án của mình. Sau đó, họ cần chọn một vòng đời phát triển sản phẩm phù hợp và thiết lập tất cả các chi tiết của dự án của họ xung quanh vòng đời đó. Việc đánh giá cấu trúc logic của các dự án sẽ do các cố vấn dự án trực tiếp thực hiện trong suốt thời gian học của họ. Đối với điều này, chúng tôi đã chỉ định các giảng viên giàu kinh nghiệm của mình thực hiện đánh giá này.

Tiêu chí 3. Hiệu quả thiết kế:

Thiết kế là một thành phần quan trọng của bất kỳ dự án EEE nào, và các cố vấn của mỗi dự án sẽ đồng hành cùng sinh viên của họ trong giai đoạn thiết kế. Các câu hỏi điển 
hình về chi phí thiết kế mới là bao nhiêu, thiết kế tiết kiệm bao nhiêu năng lượng, cách tích hợp thiết kế mới với thiết kế khác, v.v. nên nằm trong danh sách kiểm tra của tất cả các đánh giá thiết kế. Tuy nhiên, không phải người cố vấn nào cũng nắm vững các kỹ năng và kiến thức trong các khía cạnh khác nhau của thiết kế bộ điều khiển công suất, thiết kế mạch công nghiệp hoặc thiết kế mạng cảm biến, v.v.; Do đó, chúng tôi đã rất nỗ lực để kết nối chặt chẽ các thành viên trong khoa với nhau để tham khảo ý kiến lẫn nhau bất cứ khi nào. Trong thực tế, điều này cần sự nỗ lực của tất cả các giảng viên CDIO.

Tiêu chí 4. Khả năng Thị trường (của Sản phẩm hoặc Dịch vụ):

Để đánh giá khả năng bán ra thị trường của một số sản phẩm hoặc nguyên mẫu của sản phẩm dành cho sinh viên là một quá trình dài ngay cả đối với Ban Đánh giá CDIO của chúng tôi. Mặc dù người cố vấn có thể đưa ra bất kỳ đánh giá và phân loại nào về tiếp thị của sản phẩm và nguyên mẫu sản phẩm, nhưng chỉ một số rất ít dự án được đánh giá là đặc biệt. Mỗi giảng viên và sinh viên sẽ có cơ hội đánh giá tính khả thi của dự án này như một phần của đồ án. Ngoài ra, đối với CDIO 5 chúng tôi còn đánh giá dự án theo nhận xét của khách hàng và doanh thu hoặc lợi nhuận.

Theo mô hình CDIO truyền thống, Tiêu chí 1 sẽ được đánh giá trong quá trình hình thành, Tiêu chí 2 sẽ được đánh giá trong giai đoạn thiết kế và thực hiện, và Tiêu chí 3 sẽ được đánh giá trong giai đoạn thiết kế; Nhưng trong phạm vi của mô hình CL CDIO mới của chúng tôi, tất cả các mục Tiêu chí 1-4 sẽ được đánh giá lại toàn diện hơn trong giai đoạn được xem xét cho giai đoạn hoạt động. Các đánh giá tại thời điểm này có xu hướng chính xác hơn vì chúng tôi đã có sản phẩm mẫu hoặc sản phẩm học sinh từ giai đoạn Triển khai. Ngoài ra, chúng tôi cũng mời các chuyên gia quản lý doanh nghiệp tham gia Ban đánh giá của chúng tôi để đánh giá tính khả thi của dự án khởi nghiệp. Điều này rất hiệu quả trong việc giúp học sinh học hỏi từ phản hồi từ nhiều nguồn khác nhau, và có nhiều thời gian để sinh viên tiếp thu kiến thức mới trong quá trình này.

\section{Case study và đánh giá}

\subsection{Case study}

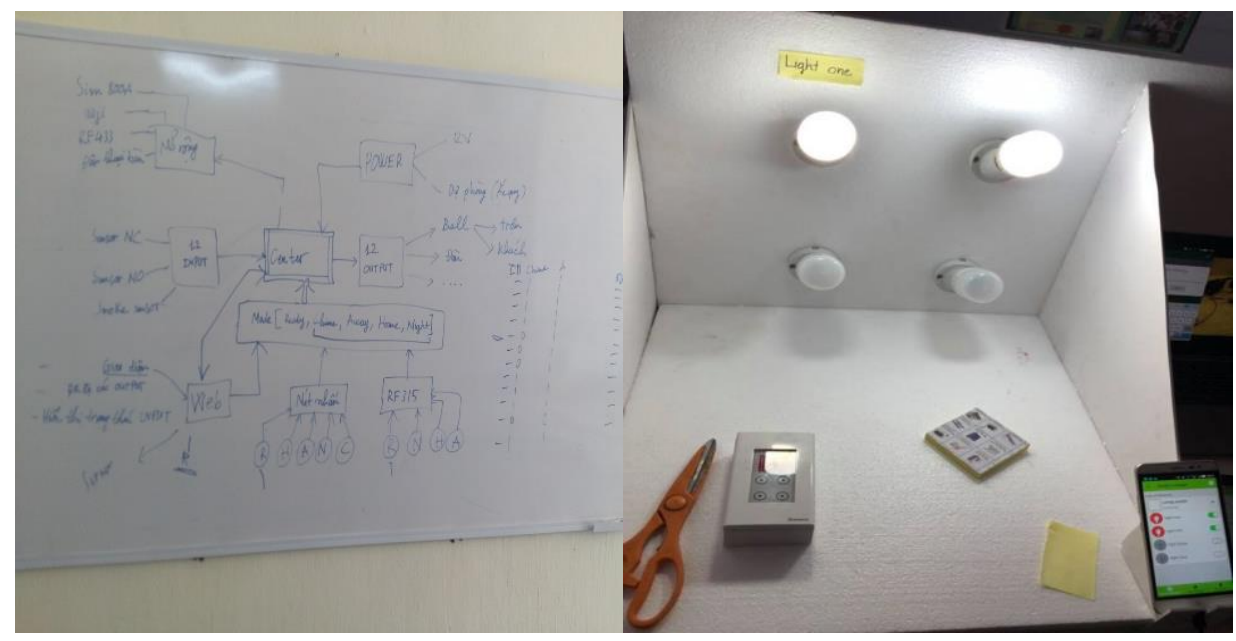

Hình 2. Một thiết kế Nhà thông minh đơn giản 
Trong phần này, chúng tôi trình bày một ví dụ để giải thích quá trình triển khai khung CDIO khép kín. Một sản phẩm tiêu biểu của EEE trong dự án CDIO khép kín của chúng tôi là ngôi nhà thông minh được phát triển bởi một nhóm từ các sinh viên K18EVT (Mr Hà Kim Tùng, Mr Nguyễn Mạnh Tuấn và Mr Nguyễn Công Tín). Tên gọi "nhà thông minh" có nghĩa là nó là một hệ thống điện và điện tử tích hợp có thể được lập trình để điều khiển các thiết bị gia dụng như điện gia dụng, nội thất, v.v. một cách tự động hoặc theo yêu cầu của người dùng.

Trong CDIO 1, các sinh viên được yêu cầu điều tra thị trường về nhà thông minh. Tiếp theo, họ đề xuất ý tưởng trong nhóm của mình sau khi họ biết về sản phẩm nhà thông minh trên thị trường. Và họ thảo luận để đưa ra quyết định về ý tưởng về nhà thông minh. Sau đó, họ thiết kế và xây dựng một nguyên mẫu đơn giản để giới thiệu với khách hàng tiềm năng của họ, như Hình 2. Cuối cùng, họ điều chỉnh ý tưởng của mình theo những nhận xét mà họ nhận được từ cuộc khảo sát.

Trong CDIO 2, các nhóm được chọn tập trung vào thiết kế sản phẩm thực. Họ đã tính đến hình thức bên ngoài, giá thành, sự tiện lợi khi sử dụng để đáp ứng các yêu cầu của thị trường. Họ hoàn thành sản phẩm của mình bằng cách tạo mẫu thử nghiệm phiên bản thứ 2 như Hình 3 . Họ đã điều tra thị trường bằng cách hỏi một số khách hàng về thiết kế sản phẩm của họ và thảo luận trong nhóm để tối ưu hóa thiết kế của họ.

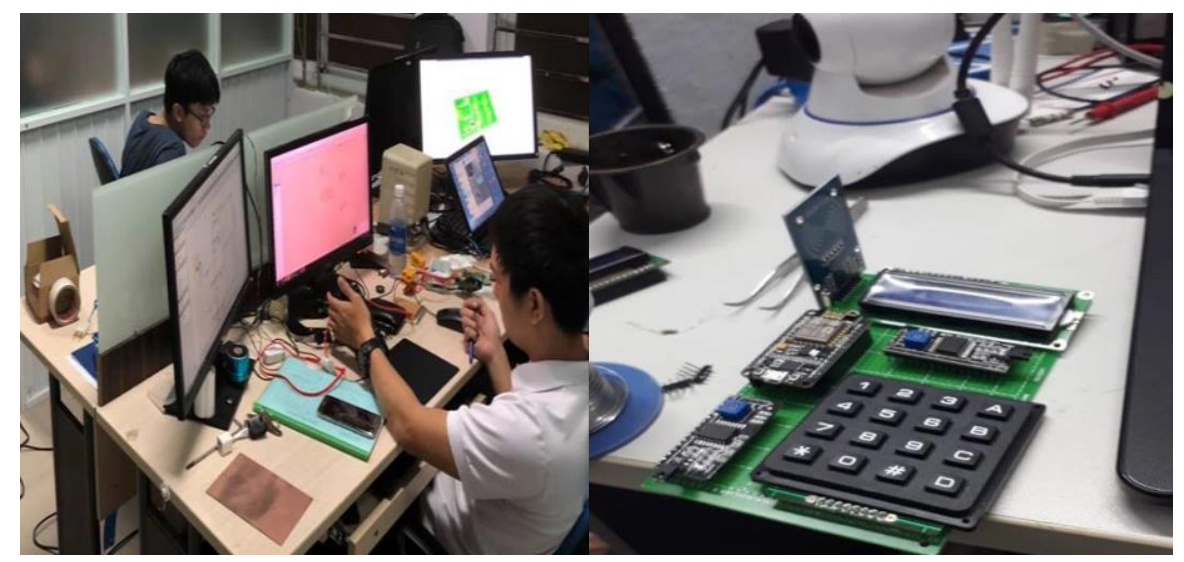

Hình 3. Phiên bản cải thiện của Nhà thông minh

Trong CDIO 3, nhóm được chọn đã lắp đặt sản phẩm nhà thông minh của họ ở vị trí của khách hàng như Hình 4 . Nhóm đã thiết kế quy trình sản xuất và lắp đặt, sau đó triển khai cài đặt sản phẩm cho ngôi nhà của người dùng. Cuối cùng, họ đánh giá hoạt động của sản phẩm dựa trên kết quả đo lường và điều tra của người dùng.

Trong CDIO 4, nhóm này tiếp tục đề xuất các ý tưởng để cải thiện hoạt động đồng thời duy trì hoạt động của sản phẩm của họ ở vị trí của khách hàng bằng cách thêm một chức năng mới theo yêu cầu của khách hàng. Hình 4 mô tả việc lắp đặt hệ thống điều khiển tích hợp với hệ thống nhà thông minh. Cuối cùng, họ đánh giá hoạt động của sản phẩm dựa trên kết quả đo lường và điều tra của người dùng.

Trong CDIO 5, nhóm được xem xét này đã thành lập một công ty, đó là Công ty TNHH Efil (https://doanhnghiepmoi.vn/thong-tin/CONG-TY-TNHH-EFIL-47393.html), để điều hành dự án kinh doanh của dòng sản phẩm này. Các cố vấn đến từ Khoa Quản lý Kinh doanh cũng giúp họ bằng cách cung cấp một số kiến thức trong lĩnh vực kinh doanh. Ngoài ra, họ cũng đã 
tham gia vườn ươm doanh nghiệp Đà Nẵng (https://www.linkedin.com/company/danangbusiness-incubator) để được chính quyền thành phố Đà Nẵng hỗ trợ nhiều hơn.

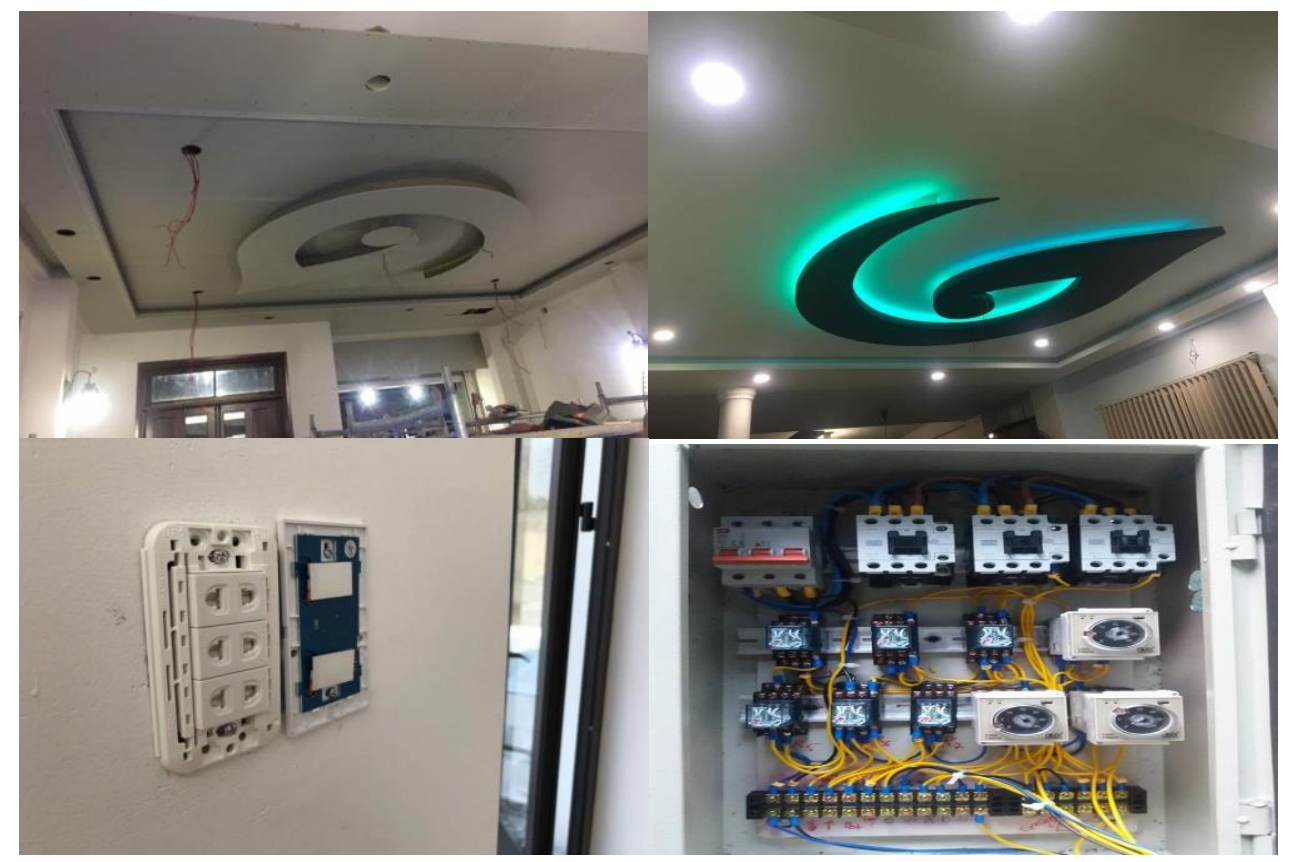

Hình 4. Lắp đặt hệ thống nhà thông minh trên thực tế

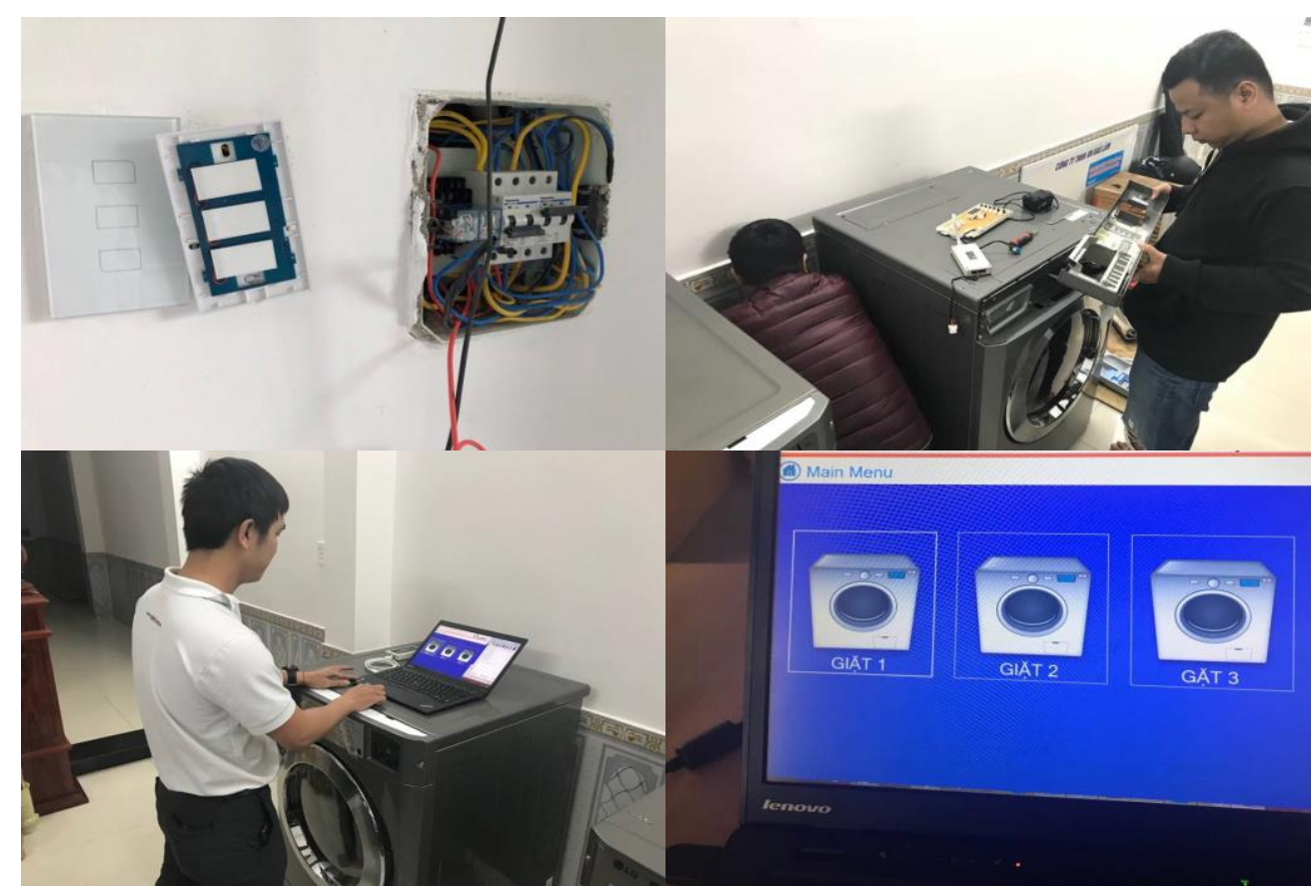

Hinh 5. Cải tiến và hoàn thiện Nhà thông minh

\section{2. Đánh giá}

Trong quá trình áp dụng mô hình CDIO khép kín tại Khoa Điện-Điện tử, Đại học Duy Tân, chúng tôi đánh giá hiệu quả của mô hình này dựa trên tinh thần khởi nghiệp của sinh viên thông qua các dự án kinh doanh. Tuy nhiên, mô hình đề xuất này có những ưu và nhược điểm sau: 
U’u điểm:

$\checkmark$ Có thể nuôi dưỡng tinh thần kinh doanh của sinh viên.

$\checkmark$ Tạo cho sinh viên thói quen không ngừng đổi mới và hoàn thiện sản phẩm.

$\checkmark$ Tạo cho sinh viên thói quen kiên trì, không bỏ cuộc.

Nhược điểm:

$\checkmark$ Khó thực hiện do kiến thức và kỹ năng trong lĩnh vực kinh doanh.

$\checkmark$ Yêu cầu thực hiện dự án trong cuộc sống thực.

$\checkmark$ Liên quan đến nhiều vấn đề khó khăn như an toàn, tiền bạc, luật pháp.

\section{Kết luận}

Trong bài báo này, chúng tôi đã trình bày một mô hình CDIO được cải thiện, cụ thể là khung CDIO khép kín. Hiệu quả của khung này đã được kiểm chứng bằng một số kết quả thực tế trong quá trình triển khai tại Đại học Duy Tân. Kết quả cho thấy mô hình CDIO khép kín được đề xuất này có thể giúp sinh viên nâng cao khả năng khởi nghiệp. Chúng tôi sẽ tiếp tục triển khai mô hình này trong trường đại học của chúng tôi để có thêm hiểu biết và lợi ích của mô hình này.

\section{Tài liệu tham khảo}

[1] Adam Szirmai, Wim Naude, Micheline Goedhuys (2011). "Entrepreneurship, Innovation, and Economic Development: An Overview”. Oxford Scholarship Online, September 2011.

[2] Fei, Bian (2013). "Study on Countermeasures for Cultivating Innovation Ability of College \& University Students". 2013 International Conference on Innovation, Management and Technology Research (ICIMTR 2013), 22-23 September, 2013, Malaysia, pp. 213-217.

[3] Wang, Yangqin et al (2013). "Innovation Ability Cultivation of Automation Major Students". International C3onference on Education Technology and Information System (ICETIS 2013), 21-22 June 2013, Sanya, China, pp. 971-974.

[4] Wu, Tong Q., Xu, Nian C., Huang, Lin Q. (2013). “Research Experimental Teaching System Based on Innovative Practice Ability”. Advanced Materials Research, Vol. 671-674, pp. 3248-3252.

[5] Zhang, Yong et al (2013). "Review on Innovative Practice Teaching and Its Quality Evaluation System for Software Engineering Specialty”. Advanced Technology in Teaching, Advances in Intelligent and Soft Computing Volume 163, pp. 389-397.

[6] Binh Dac Ha, Dong T Tran, Bao N Le (2017). “A proposal of the c-d-i-o model to improve students' capability”. 2017 13th International CDIO Conference in Calgary, Canada, pp.415-423.

[7] https://doanhnghiepmoi.vn/thong-tin/cong-ty-tnhh-efil-47393.html

[8] https://www.linkedin.com/company/danang-business-incubator

[9] https://www.facebook.com/danangstartup/?rc=p 